\title{
Choking deaths: the role of antipsychotic
}

\section{medication}

\author{
D. RUSCHENA, P. E. MULLEN, S. PALMER, P. BURGESS, S. M. CORDNER, \\ O. H. DRUMMER, C. WALLACE and J. BARRY-WALSH
}

\author{
Background An increased risk of \\ choking associated with antipsychotic \\ medication has been repeatedly \\ postulated.
}

\begin{abstract}
Aims To examine this association in a large number of cases of choking deaths.
\end{abstract}

Method Cases of individuals who had died because of choking were linked with a case register recording contacts with public mental health services. The actual and expected rates of psychiatric disorder and the presence of psychotropic medication in post-mortem blood samples were compared.

Results The 70 people who had choked to death were over 20 times more likely to have been treated previously for schizophrenia. They were also more likely to have had a prior organic psychiatric syndrome. The risk for those receiving thioridazine or lithium was, respectively, 92 times and 30 times greater than expected. Other antipsychotic and psychotropic drugs were not overrepresented.

Conclusions The increased risk of death in people with schizophrenia may be a combination of inherent predispositions and the use of specific antipsychotic drugs. The increased risk of choking in those with organic psychiatric syndromes is consistent with the consequences of compromised neurological competence.

Declaration of interest None.
Choking is one of the mechanisms postulated to connect the use of antipsychotic medication with sudden death (Hollister, 1957; Hussar, 1962; Flaherty \& Lahmeyer, 1978; Craig, 1980; Lereya et al, 1995). In this paper, 'choking' refers to solid gastric contents blocking the trachea or bronchi (a form of aspiration), or a food bolus lodging in the larynx or laryngopharynx (the 'café coronary': see Haugen, 1963). Such deaths occur in the general population, particularly in those who spend considerable periods immobile (Cameron $\&$ Zuidema, 1972). The high incidence of tachyphagia among patients with schizophrenia has been noted (Simpson et al, 1987), as has the frequent abnormality of such patients' swallowing mechanisms (Hussar \& Bragg, 1969). This could predispose individuals with schizophrenia to choking, independent of any role antipsychotic medication might play. This study examines the postulated association between death from choking and the use of psychotropic drugs, in light of the possible confounding association between choking and the presence of schizophrenic disorders.

\section{METHOD}

In this study, which was approved by the ethics committee of the Department of Human Services, Victoria, Australia, a matching survey linked the register of deaths reported to the State Coroner of Victoria with the Victorian Psychiatric Case Register (VPCR). The history and characteristics of these registers have been described elsewhere (Ruschena et al, 1998). All deaths in individuals aged 15 years and over, occurring between 1989 and 1995 , ascribed by the forensic pathologist after post-mortem examination to all forms of asphyxia were investigated. A total of 186 deaths were identified. Excluded from the sample were deaths due to strangulation (manual or by ligature); plastic bag asphyxia $(n=72)$; postural asphyxia $(n=13)$; autoerotic asphyxiation $(n=2)$; and asphyxiation by foreign substances other than food (e.g. smoke, carbon monoxide, spoons or surgical gloves; $n=4$ ). Also excluded were cases where the blood alcohol content (BAC) exceeded $0.1 \%$ $(n=25)$, including 4 cases with $\mathrm{BAC}=0.1-$ $0.2 \%$ and 10 cases with $\mathrm{BAC}=0.2-0.3 \%$. The threshold of $0.1 \%$ was chosen to balance the need to allow for a possible interaction between alcohol and psychotropic medication with the need to exclude those deaths with such a high BAC that alcohol alone was the most probable cause of impaired reflexes.

Prior contact with mental health services was established using the VPCR. Manual linkage was checked with a computer algorithm with better than $95 \%$ agreement. From the databases, information could be obtained on the individual's age and gender; whether the individual had attended publicly funded mental health services in Victoria; any diagnosis made; date of death; cause of death; circumstances of death; and the results of post-mortem toxicological analyses. Relative risks were calculated to express the difference between the actual and the expected incidence of deaths in the different mental disorders. Numbers of deaths per 100000 head of population per year were also calculated.

Drug consumption figures for the Australian population were derived from the defined daily dosage (DDD) figures published in the annual Australian Statistics on Medicines in the years 1990-1996 (relating to the years 1989-1995). These statistics were published in Canberra by the now defunct Australian Commonwealth Department of Health and Family Services. For a chronically administered drug, the DDD indicates how many people per 1000 of the population may have received a standard dose of such a listed medication (as defined by the DDD) daily. From the information published for the years 1989-1995, the size of the population receiving antipsychotics, antidepressants, anxiolytics and hypnotics during these years was estimated.

From this population figure comparisons could be drawn between the expected numbers of deaths in each population, given their frequency in the general population, and the actual number of individuals found by toxicological analysis to have been taking such medication at the time of death. 


\section{RESULTS}

\section{Study population}

Seventy choking deaths were identified over the 7-year period (44 males). The average population of Victoria aged over 15 years during the study period was 3500692 . This suggests an annual mortality rate of 0.286 deaths per 100000 persons (males 0.366 , females 0.209). Thirty-one people died following aspiration of gastric contents (18 males), and 39 from non-aspiration choking deaths (the 'café coronary'). The mean age at death was higher for those who died following an aspiration than following a non-aspiration choking death ( $Z$-test: $P=0.072$ ). The difference in age distributions between the aspiration and non-aspiration deaths was significant (two-sided $t$-test: $P<0.05$ ). Table 1 lists the mean and standard deviations of age for each population and the division among age groupings.

\section{Mental health}

Twenty-five of the 70 individuals had had prior contact with public sector mental health services: 10 had received a principal diagnosis of schizophrenia, 8 had an organic psychiatric disorder, and 1 had another psychiatric illness (Table 2). For the other
6 individuals no final diagnosis was recorded. The representation of people who had received treatment in the mental health services was significantly higher than expected. None of the groups differed from the general population in age or gender distribution. Of the 25 individuals who had been in contact with mental health services, 16 had died by the non-aspiration form of choking: 6 had organic disorders, 7 had schizophrenia, 1 had another psychiatric illness, and 2 had no final diagnosis. Relative risk calculations for non-aspiration deaths put the increased risk at 30 times that of the general population for those with schizophrenia (95\% CI 13.5-67.5), and 43 times that of the general population for those with an organic disorder (95\% CI 18.4-102.6).

\section{Toxicology}

Toxicological analysis was available for 21 of the 70 deaths, of which 14 tested positive for psychotropic medication. Antipsychotic agents were found in 8 of the choking deaths: thioridazine was found in 5 cases and lithium in 3 cases (on one occasion the drugs were found concurrently). Antidepressants and anxiolytics were found in 3 cases, in 2 of which the person had also taken hypnotics.

Table I Study population characteristics

\begin{tabular}{lccccccccc}
\hline Cause of death & & \multicolumn{7}{c}{ Age at death (years) } \\
\cline { 3 - 9 } & Total population & Mean & s.d. & $\leqslant 40$ & $41-50$ & $51-60$ & $61-70$ & $\geqslant 71$ \\
& $(n)$ & $($ years) & & $(n)$ & $(n)$ & $(n)$ & $(n)$ & $(n)$ \\
\hline Choking & 70 & 58.6 & 18.9 & 14 & 6 & 12 & 19 & 19 \\
Aspiration & 31 & 54.0 & 21.3 & 10 & 3 & 3 & 7 & 8 \\
Non-aspiration & 39 & $62.4^{1}$ & 16.3 & 4 & 3 & 9 & 12 & 11 \\
\hline
\end{tabular}

I. $0.1>P>0.05$.

Table 2 Deaths by choking: breakdown by diagnosis

\section{DISCUSSION}

Deaths due to choking, whether by aspiration or on a bolus of food, are relatively rare occurrences. Previous studies attempting to document and compare the rate of choking death for those in hospital with that of the general population have been hamstrung by small numbers. Data have also been lacking to determine mortality zine was detected were recorded on the VPCR. Three were recorded with a diagnosis of schizophrenia, one had an organic diagnosis cation taken in two cases; in the other cases it was taken with one other drug - lithium, the antidepressant dothiepin, and diazepam, respectively. Lithium was taken once each with thioridazine, dothiepin and diazepam. In the five deaths where diazepam, or dothiepin was found in thioridazine or lithium.

Table 3 documents the results of relative risk calculations that follow the population estimates provided by the DDD information. Relative risk calculations were made on the assumption that the case popuwas the total number of cases originchoking) (that is, all 70 deaths due to toxicology data were available. The use of thioridazine was associated with an increase in the relative risk of deaths due to choking to over 90 times that of the general population. Lithium was also assowith a statistically significan relative risk more than 30 times that of the general population.

\begin{tabular}{|c|c|c|c|c|c|c|c|c|}
\hline & \multirow[b]{2}{*}{$\begin{array}{l}\text { Population size } \\
\qquad \text { (n) }\end{array}$} & \multicolumn{3}{|c|}{ Deaths by choking } & \multirow[b]{2}{*}{$\begin{array}{c}\text { Mean age } \\
\text { (years) }\end{array}$} & \multirow[b]{2}{*}{$\begin{array}{l}\text { Relative } \\
\text { risk }\end{array}$} & \multirow[b]{2}{*}{$95 \% \mathrm{Cl}$} & \multirow[b]{2}{*}{$\begin{array}{l}\text { Deaths per } \\
100000 \text { per year }\end{array}$} \\
\hline & & $\begin{array}{c}\text { Total } \\
(n)\end{array}$ & $\begin{array}{c}\text { Male } \\
(n)\end{array}$ & $\begin{array}{l}\text { Female } \\
(n)\end{array}$ & & & & \\
\hline Organic psychiatric disorders & 14600 & 8 & 5 & 3 & 60.1 & $30.8^{*}$ & $14.8-64.1$ & 7.8 \\
\hline Schizophrenia & 25202 & 10 & 6 & 4 & 61.0 & $23.0^{*}$ & $11.9-44.6$ & 5.7 \\
\hline Other psychiatric illness & 73267 & 1 & - & 1 & 50.0 & 0.7 & $0.1-4.8$ & 0.2 \\
\hline No final diagnosis & 22933 & 6 & 4 & 2 & 67.5 & $14.2^{*}$ & $6.2-32.7$ & 3.7 \\
\hline Residual population & 3364690 & 45 & 29 & 16 & 56.9 & & & 0.2 \\
\hline Total population & 3500692 & 70 & 44 & 26 & 58.6 & & & 0.3 \\
\hline
\end{tabular}

$* P<0.001$. 
Table 3 Relative risk calculations for populations taking prescribed medication

\begin{tabular}{lcccc}
\hline Drug & $\begin{array}{c}\text { Average estimated } \\
\text { population }(n)\end{array}$ & $\begin{array}{c}\text { Choking deaths } \\
(n)\end{array}$ & Relative risk & $95 \% \mathrm{Cl}$ \\
\hline Thioridazine & 2925 & 5 & $92.1^{*}$ & $37.2-228.5$ \\
Lithium & 5025 & 3 & $31.2^{*}$ & $9.8-99.0$ \\
Antidepressants & 55355 & 3 & 2.8 & $0.9-8.6$ \\
Anxiolytics & 58063 & 3 & 2.7 & $0.8-8.4$ \\
Hypnotics & 62263 & 2 & 1.7 & $0.4-6.6$ \\
\hline
\end{tabular}

$* \mathrm{P}<0.001$.

rates for choking deaths in the general population (Craig, 1980). The purpose of this study was to examine the presence of choking deaths, within a large population, over a sufficiently long period to capture an adequate number of cases for meaningful analysis. This study documented an increased risk of choking deaths in association with mental illness in general and schizophrenia in particular, as well as with the use of thioridazine. This finding accords with the cohort study by Mortensen \& Juel (1990) of patients with schizophrenia, which found a significantly increased mortality due to aspiration of food.

Polypharmacy does not appear to be relevant to the increased mortality rates. Of the five deaths associated with thioridazine, in only two was the patient taking another psychotropic drug. This avoids the possible complicating factor of outright toxicity noted by Jusic \& Lader (1994). However, regular toxicological analyses search primarily for chlorpromazine, haloperidol and thioridazine. Unrecognised polypharmacy might have occurred with drugs that were not detected, such as fluphenazine.

In Victoria the law mandates the reporting to the coroner of any death that appears to have been unexpected, unnatural or to have resulted, directly or indirectly, from accident or injury. The law also mandates reporting of patients who die while held involuntarily in the care of a mental health service. In general, however, it is likely that the deaths of individuals who die at home are reported less frequently than those dying in care. Sudden death could be attributed to some natural disease process. Choking deaths, if observed, are sudden and dramatic, and their common association with active eating is such that a report of the death is made to the coroner. Calculations reveal that, given the deaths recorded on the coroner's database, for the population with schizophrenia not to have a significantly increased risk of choking death, there would have to have been 756 choking deaths. In effect, 686 such deaths would have to have gone unreported. For the population with an organic psychiatric disorder, 973 hypothetical deaths are required, and for the thioridazine risk not to be significant, the figure is 2552 deaths. It seems unlikely that such a high number of choking deaths would go unreported.

Antipsychotic medications have also been implicated in sudden cardiac deaths (Mehtonen et al, 1991; Reilly et al, 2002) and sudden cardiac deaths also obviously occur in the general community. A sudden cardiac death that appears to be due to choking therefore exists as a confounding factor that would increase the number of deaths attributed to choking.

\section{Limitations}

The VPCR does not record individuals who have received all their treatment from private practitioners. However, the vast majority of those with schizophrenia appear on the register. The VPCR population with a principal diagnosis of schizophrenia amounts to $0.7 \%$ of the total population of Victoria, equal in magnitude to the expected prevalence of schizophrenia in the general community.

In matching surveys such as this, any errors in data collection serve to decrease the chance of a match being made. Incompleteness of databases also decreases the likelihood of a match. In our survey, 12 cases were recorded on the VPCR, indicating that the individual concerned had been in contact with public sector mental health services in Victoria at some time. However, because no other information as to the outcome of their visit was available, these individuals were counted as part of the residual population.
The absence of complete toxicological information for all individuals hampers the study's capacity to differentiate between the effects of mental illness and those of the medication used to treat it. It results in a probable underestimation of the relative risk associated with the use of such drugs. An additional limitation is whether findings of 'choking' and/or 'aspiration' were made consistently to include and exclude the same phenomena. For example, in the context of food, choking may be defined to be merely the blockage of the larynx or laryngopharynx, or only appropriate where there is a complete blockage of the trachea and bronchi by an impacted food bolus. Additional studies should seek to closely monitor the definitions used for classification purposes.

\section{Findings}

Elevated rates of death due to choking have been found to be associated with a diagnosis of schizophrenia, organic disorders and the use of thioridazine. For the eight individuals with a diagnosis of an organic disorder, toxicological data were available in only one case. For the ten individuals with a diagnosis of schizophrenia, toxicological data were available in six cases: thioridazine use was detected in three of these cases, lithium in two (once used concurrently with thioridazine), temazepam alone in one case and the last case had a negative screen. For both populations the evidence is ambiguous about whether the increased rates were associated with medication, or were the result of swallowing difficulties or eating habits inherent in the illness itself (Hussar \& Bragg, 1969; Simpson et al, 1987). In fact, the possible role of tardive dyskinesia as a source of respiratory difficulty years after the discontinuance of antipsychotic therapy means that any retrospective study is limited in its capacity to determine the role of antipsychotics in choking (Yassa \& Lal, 1986).

Thirty-one of the deaths involved asphyxiation following the aspiration of food. A less dramatic aspiration could precipitate pneumonitis and aspiration pneumonia (Von Brauchitsch \& May, 1968). In the absence of adequate treatment the alveoli fill with oedematous fluid and inflammatory cells (pneumonitis), and necrosis results. Death may follow after days, weeks or months. As aspiration leading to death may be occult, and may follow 
a longer illness, it will probably not be reported to the coroner. This is especially the case given the relative infrequency with which people with schizophrenia report illness. Even if a patient's death were reported in conformity with the requirements of the governing legislation, the true cause of the pneumonia might easily be overlooked. Thus, the true extent of deaths due to aspiration that did not cause immediate asphyxiation is at this stage unknown.

\section{Clinical implications}

Aspiration may be mediated by a breakdown of the reflexes that serve to prevent oesophageal reflux, deficits in the cricopharyngeal sphincter reflexes preventing reflux of food into the pharynx, an interference with normal sweeping ciliary action in the respiratory tract, or a combination of such deficits (Plachta, 1965; Solomon, 1977). People with schizophrenia may be predisposed to choking deaths specifically by disorders of the physiology of swallowing and reflux, or by behaviour that increases the risk. Such risks are largely independent of the medications employed to treat the disorder, and in two of the deaths involving people with schizophrenia toxicological results showed they were not taking antipsychotics. The association between organic psychosyndromes and choking is not surprising as members of this group have compromised neurological competence, are often troubled by coexisting physical disorders and may have periods of reduced consciousness predisposing them to aspiration.

The strong association between thioridazine and choking deaths is as dramatic as it is unexpected. Suggestions that the risk of choking could be reduced by the co-administration of anticholinergic drugs (Simpson et al, 1987) might have led to the expectation that thioridazine would be among the safest antipsychotics, rather than uniquely associated with such deaths. We note the recent study into sudden cardiac death (Reilly et al, 2002), as well as prior studies (Mehtonen et al, 1991) that showed an increased risk associated with the use of thioridazine.

Clinically, the implications of treatment are less pronounced. Calculations to determine the number of patients that must be treated before a single choking death occurs (Cook \& Sackett, 1995) estimate that 591 patients would need to be treated with thioridazine over the 7-year period before

\section{CLINICAL IMPLICATIONS}

- The risk of choking is higher in schizophrenia and organic psychiatric illness, regardless of the medication that is administered.

- The risk of choking is relatively small, so the increased risks associated with thioridazine and lithium use must be weighed against the potential benefits of these drugs.

- Where possible, the administration of thioridazine should be accompanied by increased monitoring for the deterioration of swallowing and reflux-prevention reflexes.

\section{LIMITATIONS}

- The rare occurrence of choking deaths means that determining the magnitude of any risk found is problematic.

- The study is vulnerable to errors in data matching consequent on matching errors and incomplete data-sets.

- Incomplete toxicological information has resulted in an incomplete picture of the risks associated with specific drugs.

DAVID RUSCHENA, LLB, PAUL E. MULLEN, DSc, Department of Psychological Medicine, Monash University; SIMON PALMER, MSc, PHILIP BURGESS, PhD, Mental Health Research Institute of Victoria; STEPHEN M. CORDNER, FRCPA, OLAF H. DRUMMER, PhD, Department of Forensic Medicine, Monash University and Victorian Institute of Forensic Medicine; CAMERON WALLACE, PhD, JUSTIN BARRY-WALSH, FRANZCP, Department of Psychological Medicine, Monash University, Victoria, Australia

Correspondence: Professor Paul E. Mullen, Victorian Institute of Forensic Mental Health, Thomas Embling Hospital, Locked Bag I0, Fairfield, Victoria 3078, Australia

(First received 4 December 2002, final revision 22 May 2003, accepted I3 June 2003)

a single death occurred. For lithium, the figure is 1723 patients. This figure is based on the rate of choking in the general population. However, this study also shows increased levels of choking deaths in the population with a diagnosis of schizophrenia. If the rate of death due to choking inherent to schizophrenia is higher than that of the general population, the difference between the rates of choking with and without thioridazine would be smaller and the number of treatments needed before a death resulted would be greater.

\section{ACKNOWLEDGEMENTS}

This research was funded and encouraged by the Mental Health Branch of the Department of Human Services, Victoria. The authors thank the staff of the Victorian Institute of Forensic Medicine and the officers of the Victorian State Coroner's Office, for access to coroners' reports.

\section{REFERENCES}

Cameron, J. L. \& Zuidema, G. D. (1972) Aspiration pneumonia: magnitude and frequency of the problem. JAMA, 219, 1194-1196.

Cook, R. J. \& Sackett, D. L. (1995) The number needed to treat: a clinically useful measure of treatment effect. BMJ, 310, 452-454.

Craig, T. J. (1980) Medication use and deaths attributed to asphyxia among psychiatric patients. American Journal of Psychiatry, 137, 1366-1373.

Davis, J. M. \& Zhang, M. (1988) Sudden death in psychiatric patients. Psychiatric Annals, 18, 3II-319.

Flaherty, J. A. \& Lahmeyer, H. W. (1978) Laryngealpharyngeal dystonia as a possible cause of asphyxia with haloperidol treatment. American Journal of Psychiatry, 135, 1414-1415.

Haugen, R. K. (1963) The café coronary. JAMA, 186 142-143.

Hollister, L. E. (1957) Unexpected asphyxial deaths and tranquilizing drugs. American Journal of Psychiatry, II4, $1035-1038$. 
Hussar, A. E. (1962) Effect of tranquillisers on medical morbidity and mortality in a mental hospital. JAMA, $\mathbf{1 7 9}$ 682-686.

_ \& Bragg, D. G. (1969) The effect of chlorpromazine on the swallowing function in chronic schizophrenic patients. American Journal of Psychiatry, 126, 570-573.

Jusic, N. \& Lader, M. (1994) Post-mortem antipsychotic drug concentrations and unexplained deaths. British Journal of Psychiatry, 165, 787-791.

Lereya, J., Segal, A. \& Elizur, A. (1995) Sudden death neuroleptics and psychotic agitation. Progressive Neuropsychopharmacology and Biological Psychiatry, 19 229-241

Mehtonen, O. P., Aranko, K., Malkonen, L., et al

(1991) Survey of sudden death associated with the use of antipsychotic or antidepressant drugs: 49 cases in Finland. Acta Psychiatrica Scandinavica, 84, 58-64.

Mortensen, P. B. \& Juel, K. (1990) Mortality and causes of death in schizophrenic patients in Denmark. Acta Psychiatrica Scandinavica, 8I, 372-377.

Plachta, A. (1965) Asphyxia relatively inherent to tranquilisation. Archives of General Psychiatry, I2, I52-158.

Reilly, J. G., Ayis, S. A., Ferrier, I. N., et al (2002) Thioridazine and sudden unexplained death in psychiatric in-patients. British Journal of Psychiatry, $\mathbf{1 8 0}$ 515-522.

Ruschena, D., Mullen, P. E., Burgess, P., et al (1998) Sudden death in psychiatric patients. British journal of Psychiatry, I72, 331-336.
Simpson, G. M., Davis, J. M., Jefferson, J.W., et al (1987) Sudden Death in Psychiatric Patients: The Role of Neuroleptic Drugs. American Psychiatric Association Task Force Report 27. Washington, DC: APA

Solomon, K. (1977) Phenothiazine-induced bulbar palsy syndrome and sudden death. American Journal of Psychiatry, 134, 188

Von Brauchitsch, H. \& May, W. (1968) Deaths from aspiration and asphyxia in a mental hospital. Archives of General Psychiatry, 18, 129-136.

Yassa, R. \& Lal, S. (1986) Respiratory irregularity and tardive dyskinesia. Acta Psychiatrica Scandinavica, 73 506-510. 